of the report and a general note on research matters requiring further investigation, based on a report prepared by a working-group appointed during the symposium. The summary of section $A$ contains a useful review in tabular form of types and applications of resonance and pulse velocity tests with their advantages and disadvantages. Like most of the papers, however, this summary is more concerned with laboratory tests than with field-tests, and an important omission in regard to the latter is mention of the practical difficulty of measuring path-lengths sufficiently accurately in testing cast in-situ concrete structures by the pulse velocity method. Some of the statements in the second table of this summary should also be interpreted with reserve, particularly the point that the variability of the quality of the concrete within a test specimen or structure can be obtained using the pulse velocity method. This has not yet been satisfactorily established: the variability of the pulse velocity may indicate the variability of the concrete in a structure, since a reduction of the pulse velocity usually indicates a reduction in strength, but relationships between pulse velocity and strength are not closely definable in the field.

The first six papers, from France, South Africa, Japan, United States and Italy, describe apparatus and techniques for measuring the elastic properties of concrete by resonance tests on laboratory specimens in longitudinal and transverse vibration. The paper by N. Stutterheim, J. P. A. Lochner and J. F. Burger (South Africa), including the results of corrosion studies on concrete and cement mortar, is of particular interest, while C. E. Kesler and Y. Higuchi (United States) also contribute a useful paper on experimental problems. The remaining seven papers on resonance tests, from Japan, France, Switzerland and Great Britain, consider the interpretation of test data. A topic common to the majority of these papers is the relationship between the elastic properties and the strength of concrete. The dynamic and static moduli of concrete are compared by J. P. Daxelhofer (Switzerland), R. H. Elvery (Great Britain) and A. Lazard (France), and further remarks on this matter are included by $\mathbf{R}$. Cabarat (France), T. Takabayashi (Japan) and F. Arredi (Italy). Daxelhofer's contribution includes observations on the use of resonance tests in studying frost damage to concrete at the Lausanne Polytechnic.

The succeeding thirteen papers of section $A$ are concerned with pulse velocity measurements and their interpretation. Relationships between pulse velocity and the strength of concrete are dealt with by R. Jonos (Great Britain), J. Andersen and P. Nerenst (Denmark), Y. Kameda, K. Awaya and I. Yokoyama (Japan), G. Magnel and G. Huyghe (Belgium) and M. Okushima and Y. Kosaka (Japan), while some information on relationships for stones is given by M. Mamillan. Jones's paper is noteworthy as a summary of much basic research at the Road Research Laboratory, Harmondsworth, and Andersen and Nerenst give a detailed account of their experience using the condenser chronograph developed at the State Testing Laboratory, Copenhagen. These two papers and those by Kameda, Awaya and Yokoyama, by A. Voellmy (Switzerland) and by Magnel and Huyghe, will probably be the papers of most interest to readers concerned with field-work on concrete, and a paper by G. Dawance to readers concerned with field-measurements on rocks. A section in the paper by J. Chefdeville (France) on the detection of faults and cracks supplements Jones's remarks on this subject, and the detection of fire damage is considered by Andersen and Nerenst and in the joint paper by Kameda and his colleagues.

In section $B$, on hardness testing, papers are contributed from Yugoslavia, Germany, Switzerland, Brazil and France, in which countries hardness tests on concrete have received greatest attention. K. Gaede (Germany) and E. Schmidt (Switzerland), prominent workers in this field, present papers describing the use of the Franck, Einbeck and Schmidt hammers, while G. Weil (Germany) points out that the Franck hammer is favoured in Stuttgart. Papers by G. Dawance and D. Jetvic, R. Peltier and P. Meynier describe experience of the use of the Schmidt hammer in France, and P. Vassitch (Yugoslavia) discusses general problems associated with hardness tests and considers both indentation and rebound measurements. A. Voellmy compares results obtained using different types of hammer and by penetration tests, and implies that the Schmidt hammer is preferable to other types. This point is clearly a matter of controversy among workers with experience of hardness testing, however.

The four papers of section $C$ describe radiographic testing techniques that are still in their infancy. With one exception-a short note by G. Huyghe (Belgium) - they deal with research in France. J. Brochard describes and discusses density measurements by gamma-radiation at the Centre Expérimentale de Recherches et d'Études du Bâtimeat et des Travaux Publics and the measurement of water content by neutron bombardment. J. P. Fackler's paper is a detailed account of gamma-ray tests on concrete at the Centre Scientifique et Technique du Bâtiment for the specialist, and J. Jacquesson's paper considers a problem in the technique of measuring soil densities.

The report is a useful and informative account of progress and investigations prior to 1954. It appears that it was decided to omit some of the discussion in the report, presumably in view of the large number of papers. Presentation of a large number of papers during the symposium had the effect of reducing the time spent on discussing the difficulties attending application of the methods of test and the practical significance of field-test results obtained by them. These matters are vitally important, and determine the value and promise of existing techniques of nondestructive testing in regard to concrete construction.

A. E. SEDDON

\section{TEXTILE INDUSTRIES IN THE UNIVERSITY OF LEEDS}

\section{REPORT FOR 1953-54}

$\mathrm{T}$ HE eightieth report, to the Worshipful Company of Clothworkers of the City of London, of the Advisory Committee of the Departments of Textile Industries and Colour Chemistry and Dyoing, University of Lecds*, covering the session 1953-54, notes that the new building to house the man-made fibres division of the Department was rapidly approaching

* Report to the Worshipful Company of Clothworkers of the City of London of the Advisory Committee on the Departments of Textile Industries and Colour Chemistry and Dyeing in the University of
I.eeds, for the Session 1953-54. $\mathrm{Pp}$. 42. (Leeds : The University, 1955.) 
completion. A third chair, that of textile technology, has now been instituted in the Department, and the research school in the Department of Colour Chemistry and Dyeing has been further strengthened by the appointment of an Imperial Chemical Industries, Ltd., Research Fellow. The graduates of both Depart. ments are in keen demand by many branches of industry. In textile physics further work has been carried out on the examination of fibre surfaces by replica methods, and a study of the fine structure of keratin fibres disintegrated by various techniques has shown that super-contraction is molecular rather than fibrillar in origin. A study has been made of the pigment granules isolated from various wools and hairs, and of their location in the fibres. In textile chemistry good progress is reported in the synthesis of polypeptides and related compounds, and in the search for new cross-linking agents it was found that reductone undergoes a unique type of polymerization in aqueous solution and that the polymer so formed is deposited inside the fibres. Further work was carried out on the initiation of the polymerization of vinyl compounds inside wool fibres with persulphates, on the formation of polyacrylonitrile in wool and on suint and wool wax ; a strikingly novel approach has been developed to the problem of dimensional stability, and a mixture of fluorescent dyes has been devised which gives different colours with wool, cellulosic fibres, cellulose acetate, nylon, 'Terylene' and 'Orlon'. In textile engineering, work continued on the development of the new type of cap designed to give uniform spinning tension, on the drying of wet fibrous materials and theoretical studies on the use of felts in removing water in the manufacture of paper. In textile technology the electronic mule was being used with great success for studying the behaviour of fibres during the drafting of condensed slivers, and a comprehensive investigation was in progress on the effects of weaving tensions on cloth characteristics with special reference to rayon fabrics. Changes occurring in the setting of nylon were being studied, and further progress has been made in work on the proofing of viscose rayon sgainst bacterial attack.

The Department of Colour Chemistry and Dyeing made further progress in its studies of the basic reactions of anthraquinone chemistry, completing the direct hydroxylation of peri-naphthalimide and the quinoxaline derived from acenaphthenequinone, and pyranthrone, amphi-isopyranthrone, and 6-phenylmesobenzanthrone. Other work has related to the mode of formation of the anthraquinone carbazole dyes from derivatives of 1 : $I^{\prime}$-dianthraquinonylamine, the reactions involved in the reconversion of sulphuric esters of reduced forms of vat dyes into the parent compounds, and on the nature of the products formed by the 'over-reduction' of indanthrone. Work on the action of Grignard reagents and dialkylcadmiums on alkylquinolinium salts was completed, and a relation found between the basic strength of anions and their orientation in nuclear substitution reactions. Work on the properties of the $\mathrm{N}$-methyl-derivatives of anthraquinone-acridine dyes, the chemistry and dyeing properties of hæmatin, the colouring matter of logwood, and quantitative studies on the dyeing characteristics of chlorinated wool continued, while the dyeing of blends of wool and 'Fibrolane', and the dyeing characteristics of wool-nylon, wool-'Ardil' and nylon-'Ardil' for mixtures of acid and pre-metallized acid dyes were also investigated. Lists of publications are included.

\section{HIGH-ENERGY PARTICLE ACCELERATORS IN THE UNITED STATES}

ONE of the most noteworthy features of the International Conference on the Peaceful Uses of Atomic Energy held in Geneva last August was the series of evening lectures delivered by distinguished scientists. In particular, Prof. E. O. Lawrence's talk on August 11, in which he outlined the research programme in the United States concerned with the production of large currents of highenergy sub-atomic particles by means of accelerators, was especially interesting and provoked much dis. cussion. The illustrated article, "High-Current Accelerators", which is contributed by Prof. Lawrence to the December 9 issue of Science $(122,1127$; 1955), is based on his talk to the Geneva conference, and in it he emphasizes the importance of the problem of the production of intense beams of high-energy particles for future progress in nuclear physics. This is illustrated by the remark that the particle current has steadily decreased as the energy has increasedmilliamperes for the 194.0 cyclotron, microamperes for the 1943 synchrocyclotron, millimicroamperes for the present cosmotron and bevatron-and that, extrapolating on this basis, currents of only two protons per hour will be produced by accelerators giving particles of $10^{16} \mathrm{eV}$. energy. Reference is made to the work of $\mathrm{L}$. H. Thomas and to the later approaches of V. Veksler and E. M. McMillan to the problem of a suitable magnetic field for a high-energy cyclotron. The basic similarity between the solutions in providing an azimuthal variation in the magnetic field to achieve focusing of the particles is pointed out.

Prof. Lawrence then describes briefly experiments that have been carried out in the Radiation Laboratory of the University of California, Berkeley, where he is director, with an electron model of a clover-leaf (this refers to the shape of the magnet pole-pieces) cyclotron. The model operated in complete agree. ment with theoretical expectations and showed that the Thomas-type field can provide the necessary axial and radial stability and that the accelerated electrons can stay in phase with the constantfrequency accelerating voltage. In addition to producing high currents of high-energy particles, equivalent to $300-\mathrm{MeV}$. deuterons, with very low voltages on the accelerating electrodes the clover-leaf cyclotron possessed the admirable property of easy extraction of the beam. Mention is also made of recent novel approaches that have been suggested to the problem of adapting cyclotrons and betatrons to produce high currents of high-energy particles, and these are now being put to practical test by the accelerator design group of the Mid-western Universities Research Association under the direction of Prof. D. W. Kerst.

During the past four or five years, along with the development of the clover-leaf cyclotron, the Radiation Laboratory has concentrated much effort, in collaboration with the California Research and Development Co., on the construction of a linear accelerator of the cavity-resonator type devised by L. W. Alvarez. The objective is to produce a particle accelerator capable of delivering thousands of kilowatts of high-energy protons. Details are given by Prof. Lawrence in his article of this $A-48$ linear accelerator, including the ion-injector, the beam focusing and steering magnet, the 24-Mc./s. buncher 